\title{
Aerosol Synthesis and Gas-Phase Photoelectron Spectroscopy of
} Ag-Bi-I Nanosystems

Danijela Danilovićc ${ }^{[1]}$, Dušan K. Božanic ${ }^{[1],{ }^{*}, \text { Radovan Dojčilović }}{ }^{[1]}$, Nenad Vukmirovićc ${ }^{[2]}$, Pitambar Sapkota $^{[3,4]}$, Ivana Vukašinović ${ }^{[5]}$, Vladimir Djokovic ${ }^{[1]}$, John Bozek ${ }^{[6]}$, Christophe Nicolas ${ }^{[6]}$, Sylwia Ptasinska ${ }^{[3,4]}$, and Aleksandar R. Milosavljevic ${ }^{[6], *}$

${ }^{I}$ Department of Radiation Chemistry and Physics, "Vinča" Institute of Nuclear Sciences - National Institute of the Republic of Serbia, University of Belgrade, P.O. Box 522, 11001 Belgrade, Serbia

${ }^{2}$ Institute of Physics Belgrade, University of Belgrade, Pregrevica 118, 11080, Belgrade, Serbia

${ }^{3}$ Radiation Laboratory, University of Notre Dame, Notre Dame, IN 46556, USA

${ }^{4}$ Department of Physics, University of Notre Dame, Notre Dame, IN 46556, USA

${ }^{5}$ Faculty of Agriculture, University of Belgrade, Nemanjina 6, 11080 Belgrade, Serbia

${ }^{6}$ SOLEIL, l'Orme des Merisiers, St. Aubin, BP48, 91192 Gif sur Yvette Cedex, France

CorrespondingAuthors:*D.K.B.bozanic@vin.bg.ac.rs; *A.R.M.milosavljevic@synchrotron-soleil.fr 


\section{EXPERIMENTAL SECTION}

\section{Ag-Bi-I precursor solution}

The Ag-Bi-I precursor solution in dimethyl sulfoxide (DMSO) was prepared by homogenization of $234 \mathrm{mg}$ of $\mathrm{AgI}$ and $295 \mathrm{mg}$ of $\mathrm{BiI}_{3}$ in mortar and subsequent dispersion of the homogenized powder in $50 \mathrm{ml}$ of DMSO. The resulting of $1.058 \mathrm{wt} / \mathrm{vol} \%$ precursor solution was clear and without precipitate, as it is shown in Figure S1.

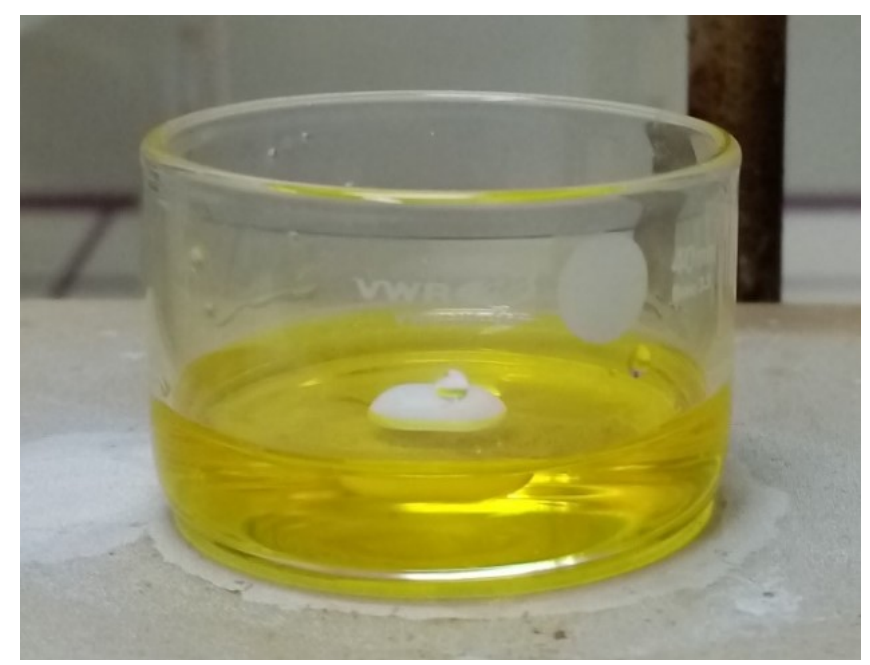

Figure S1. Photograph of 1.058 wt./vol.\% AgI/BiI 3 precursor solution in DMSO.

\section{Aerosol generation and X-ray aerosol photoelectron spectroscopy (XAPS)}

The system for Ag-Bi-I aerosols generation and X-ray aerosol photoelectron spectroscopy (XAPS) is schematically presented in Figure S2. The aerosols were generated by atomization of the precursor solution using TSI 3076 atomizer, then transported using 2.5 bar of either Ar or $\mathrm{N}_{2}$ carrier gas (depending on the desired photoelectron kinetic energy range) first through a metallic tube (heated to a temperature of about $120^{\circ} \mathrm{C}$ to evaporate DMSO), and then through a cold trap (cooled to $0{ }^{\circ} \mathrm{C}$ to remove excess DMSO from the beam).

XAPS was performed at the PLEIADES beamline at SOLEIL synchrotron, France, using the Multi-Purpose Source Chamber (MPSC) setup described in details previously. ${ }^{1}$ After the generation of Ag-Bi-I nanoparticle (NP) aerosols and removal of excess DMSO solvent (see above), the aerosols were introduced in the MPSC differentially pumped chamber through a $200 \mu \mathrm{m}$ limiting orifice, followed by an aerodynamic lens system (ALS) that focuses the NP beam through a skimmer to the ionization chamber at $10^{-8}$ mbar base pressure $\left(10^{-6}-10^{-5}\right.$ mbar working pressure). The Ag-Bi-I NP beam was crossed by a vertically linearly 
polarized synchrotron radiation photon beam in front of an electron energy analyzer. Both the exit of ALS and the skimmer position can be remotely controlled to align the nanoparticle beam to both the photon beam and the focal point of the electron analyzer. The aerosol source conditions were stable during the experiment and XPS spectra did not show any systematic change over the time related to its parameters.

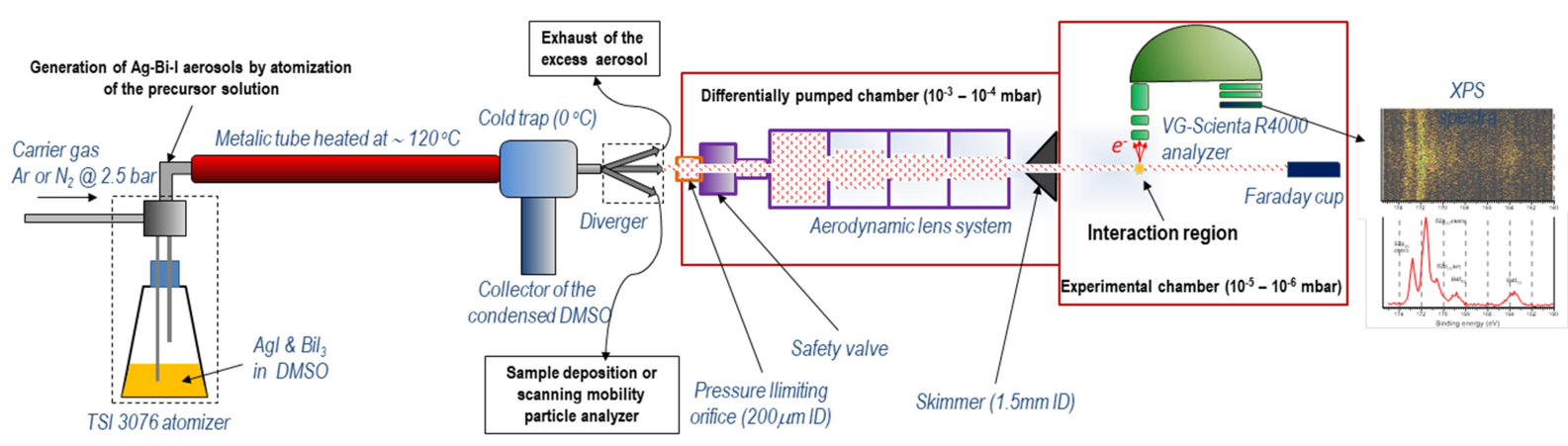

Figure S2. Schematic representation of the setup used for Ag-Bi-I aerosol generation and X-ray aerosol photoelectron spectroscopy (XAPS) at the PLEIADES beamline at SOLEIL synchrotron (France).

\section{Additional data on the characterization of $\mathrm{Ag}_{3} \mathrm{BiI}_{6}$ nanoparticles}
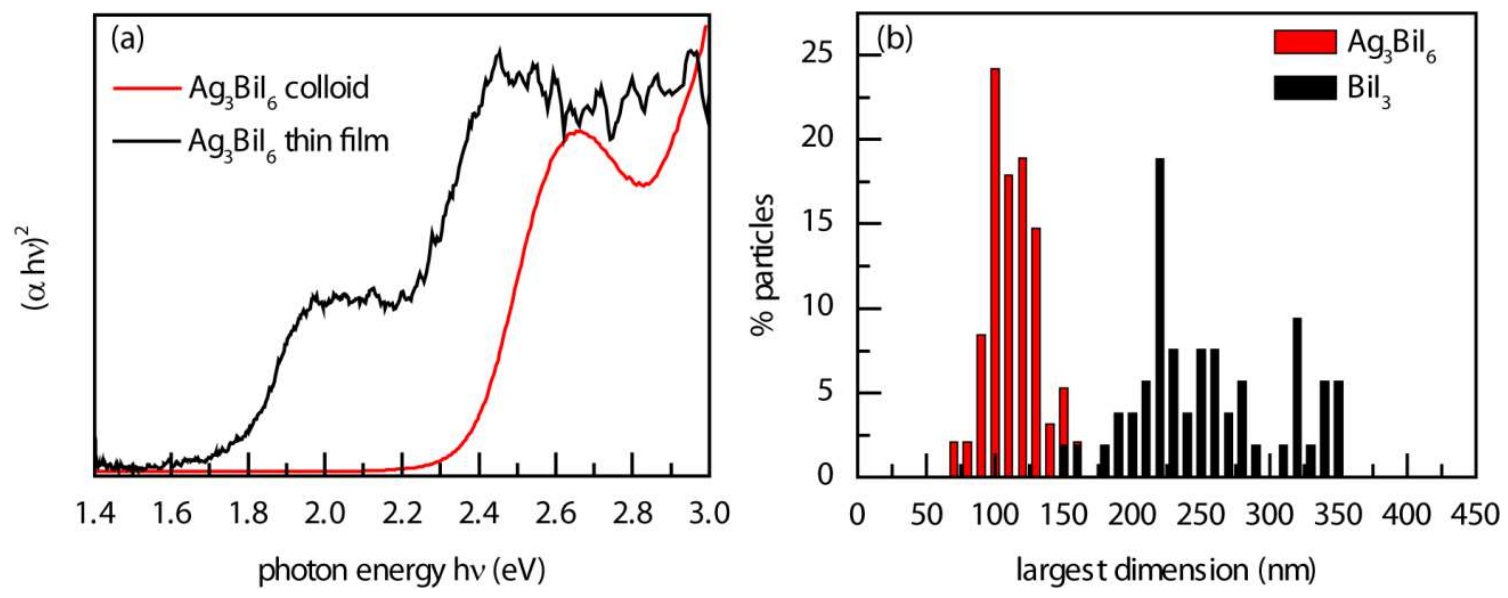

Figure S3. (a) Optical absorption spectra of $\mathrm{Ag}_{3} \mathrm{BiI}_{6}$ particles dispersed in acetonitrile (red line) and of a thin $\mathrm{Ag}_{3} \mathrm{BiI}_{6}$ film deposited on a quartz substrate (black line). (b) Distribution of the largest dimensions of the $\mathrm{Ag}_{3} \mathrm{BiI}_{6}$ and $\mathrm{BiI}_{3}$ particles observed by SEM (Figures $1 \mathrm{c}$ and $1 \mathrm{~d}$ ).

\section{XPS of nanoparticles and gas phase molecules}

The aerodynamic lens system (see Figure S2) focuses the nanoparticle (NP) beam and thus provides a high NP target density in the interaction region, leading to a sufficiently high 
photoelectron signal to noise ratio, which is necessary to perform XPS spectroscopy of desired target species. Moreover, the focused NP beam also allows to distinguish an XPS signal related to the photoionization of NPs from the one originating from small atoms/molecules such as carrier gas, residual solvent and air background.

Figure S4 schematically represents principles of the XPS spectroscopy of a NP beam using the VG-Scienta R4000 electron energy analyzer at PLEIADES beamline. After being focused by the aerodynamic lens, the NP beam enters the ionization chamber through a skimmer and crosses perpendicularly the photon beam in front of the Scienta analyzer entrance lens (not shown in the scheme). The aerodynamic lens is designed to focus large nanoparticles $(50-500 \mathrm{~nm})$, whereas small species such as carrier gas (Ar, $\mathrm{N}_{2}$, air) or residual solvent molecules $\left(\mathrm{H}_{2} \mathrm{O}\right.$, ethanol, ACN, DMSO etc.) diffuse at the exit of the lens. Therefore, majority of these molecules do not pass through the skimmer and stay in the differentially pumped MPSC chamber, resulting in a low working pressure in the ionization chamber (Figure S2). Part of the carrier gas and residual solvent still makes through the skimmer but since not focused quickly fill a large volume of the ionization chamber (Figure S4, left).

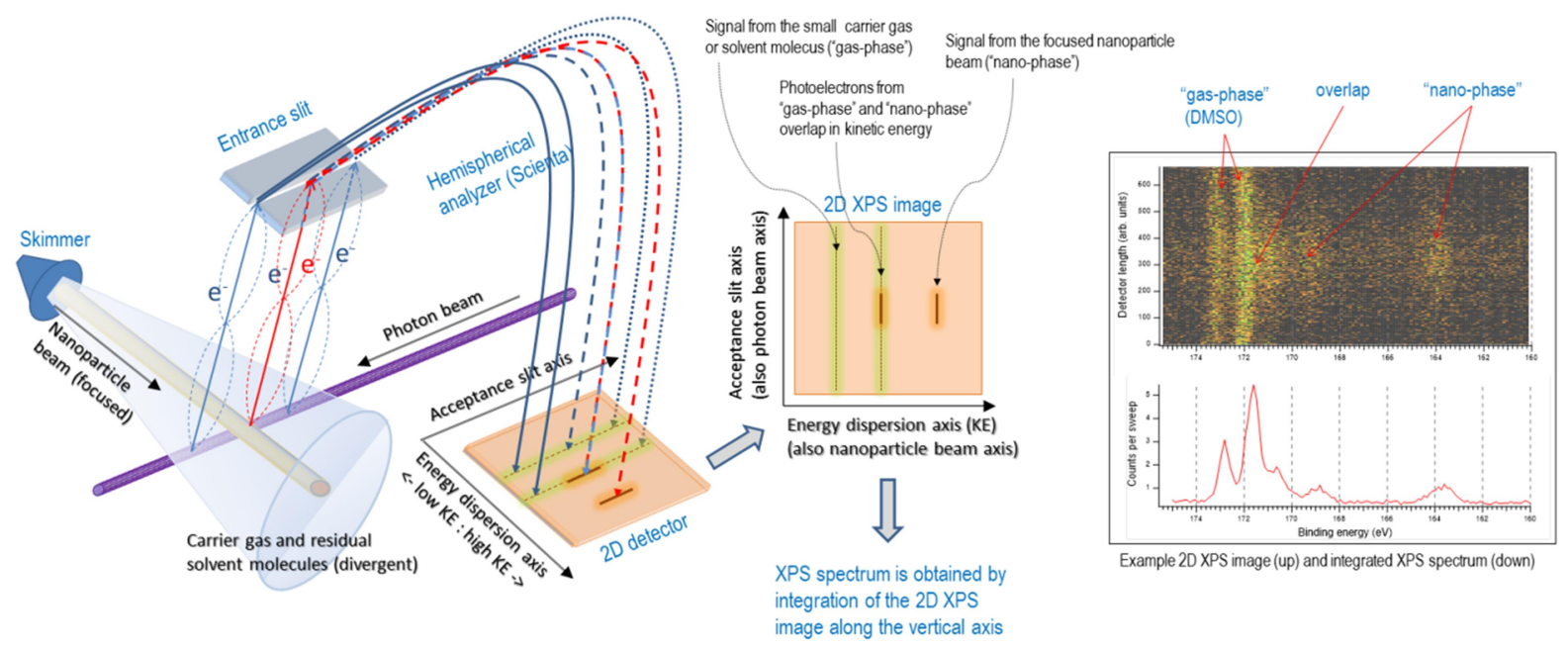

Figure S4. Schematic representation of the 2D XPS imaging of a nanoparticle beam at the PLEIADES beamline at SOLEIL synchrotron (France), as well as an example X-ray photoelectron spectrum in two-dimensions (top) and as an integrated spectrum (bottom).

The photon beam ionizes both the focused NP beam and the small molecules along its path and produces photoelectrons. The kinetic energy distribution of the photoelectrons is obtained by dispersing their trajectories inside a hemispherical analyzer, according to their kinetic energies in the horizontal direction onto a position sensitive detector, as schematically 
presented in Figure S4. Only photoelectrons produced within a limited interaction area defined roughly by the entrance slit are detected. When Scienta analyzer works in a so called "transmission mode", all electrons emitted along the axis of the ionizing photon beam are focused to the same vertical position of the detector, independently from their emission angles. Consequently, photoelectrons from the central (along the photon beam direction) part of the interaction region are imaged onto the central (along Y-axis) part of the 2D XPS image (see Figure S4, middle). Note that the axis of the electron energy dispersion (X-axis on the 2D XPS image) coincides with the axis of the nanoparticle beam direction. On the other hand, the axis of the entrance slit (Y-axis of the 2D XPS image) coincidence with the photon beam direction.

Since large NPs are focused to form a beam of sub-millimetre cross-section dimeter (typically a few hundred microns), which is markedly smaller than the Scienta entrance slit length, the photoelectrons coming from the NP beam are imaged only onto the central part (along Y-axis) of the 2D XPS image. On the other hand, the photoelectrons coming from the carrier gas and residual solvent molecules fill the whole area of entrance slit because the former are not focused thus form a diffuse cloud around the central nanoparticle beam. Therefore, photoelectron lines corresponding to the nanoparticle beam ("nano-phase") are seen in the center of the image while those from the gaseous non-focused species ("gasphase") fill the whole image, allowing the two components to be easily identified. Note also that the background is dominantly produced from dense nanoparticle beam. 


\section{THEORETICAL SECTION}

\section{Calculations based on PBE GGA functional within DFT}

Electronic structure calculations based on density functional theory (DFT) were performed. Exchange correlation functional was modelled using Perdew-Burke-Ernzerhof (PBE) generalized gradient approximation $(\mathrm{GGA}){ }^{2}$ Effects of spin-orbit interaction were fully included. The effect of core electrons was modeled using norm-conserving fully relativistic pseudopotentials. $^{3,4}$

Calculations were performed using the plane-wave code Quantum Espresso. ${ }^{5,6}$ Atomic coordinates and lattice parameters were relaxed until atomic forces were smaller than $10^{-3}$ $\mathrm{Ry} / \mathrm{bohr}$. Kinetic energy cutoff for plane-wave representation of wave functions was $40 \mathrm{Ry}$ for $\mathrm{BiI}_{3}$ and $50 \mathrm{Ry}$ for Ag-Bi-I. The structures considered and their lattice parameters obtained after relaxation are summarized in Table $\mathrm{S} 1$.

Table S1. Structures considered and their lattice parameters obtained after relaxation.

- $\mathrm{BiI}_{3}$, space group \#148

primitive cell: $2 \mathrm{Bi}$ atoms and $6 \mathrm{I}$ atoms

unit cell: 6 Bi atoms and 18 atoms

lattice parameters of hexagonal unit cell:

$a=b=7.25 \dot{A}, c=20.58 \dot{A}, \alpha=\beta=90^{\circ}, \gamma=120^{\circ}$

- $\mathrm{AgBiI}_{4}$, space group \#74

primitive cell: $2 \mathrm{Ag}, 2 \mathrm{Bi}$ and $8 \mathrm{I}$ atoms

unit cell: $4 \mathrm{Ag}, 4 \mathrm{Bi}$ and $16 \mathrm{I}$ atoms

lattice parameters of orthorhombic unit cell:

$a=8.35 \dot{A}, b=8.26 \dot{A}, c=11.93 \dot{A}, \alpha=\beta=\gamma=90^{\circ}$

To obtain the positions of band energies with respect to vacuum, we have performed electronic structure calculation of the system consisting of the interface of the material and the vacuum, see middle parts of Figures S5 and S6. In the case of $\mathrm{BiI}_{3}$ we considered the system consisting of a $\mathrm{BiI}_{3}$ slab whose width in $c$ direction is equal to $2 c$ and a vacuum slab of the same width; see middle part of Fig. S5. In the case of $\mathrm{AgBiI}_{4}$ we considered the system consisting of a vacuum slab of width $2 c$ and an $\mathrm{AgBiI}_{4}$ slab whose width in $c$ direction is equal to $2 c$, which was terminated with a surface layer containing $\mathrm{Ag}$ and I atoms; see middle part of Fig. S6.

The profile of the electronic potential (more precisely the sum of the Hartree potential and the local part of the ionic pseudopotential) in the direction perpendicular to the interface is 
presented in the top parts of Figs. S5 and S6. The potential presented in the figures was obtained by averaging in the plane parallel to the interface. The same potential profile for bulk materials is presented in the bottom part of Figs. S5 and S6. To obtain the positions of band energies with respect to vacuum, we determine the energy shift that is necessary to align the bulk potential profile with a potential profile in the material slab. We then use this information to determine the positions of bulk band energies with respect to vacuum.

We find that $\mathrm{VBM}$ for $\mathrm{AgBiI}_{4}$ and $\mathrm{BiI}_{3}$ are respectively $-5.4 \mathrm{eV}$ and $-5.9 \mathrm{eV}$ when $\mathrm{PBE}$ functional is used.
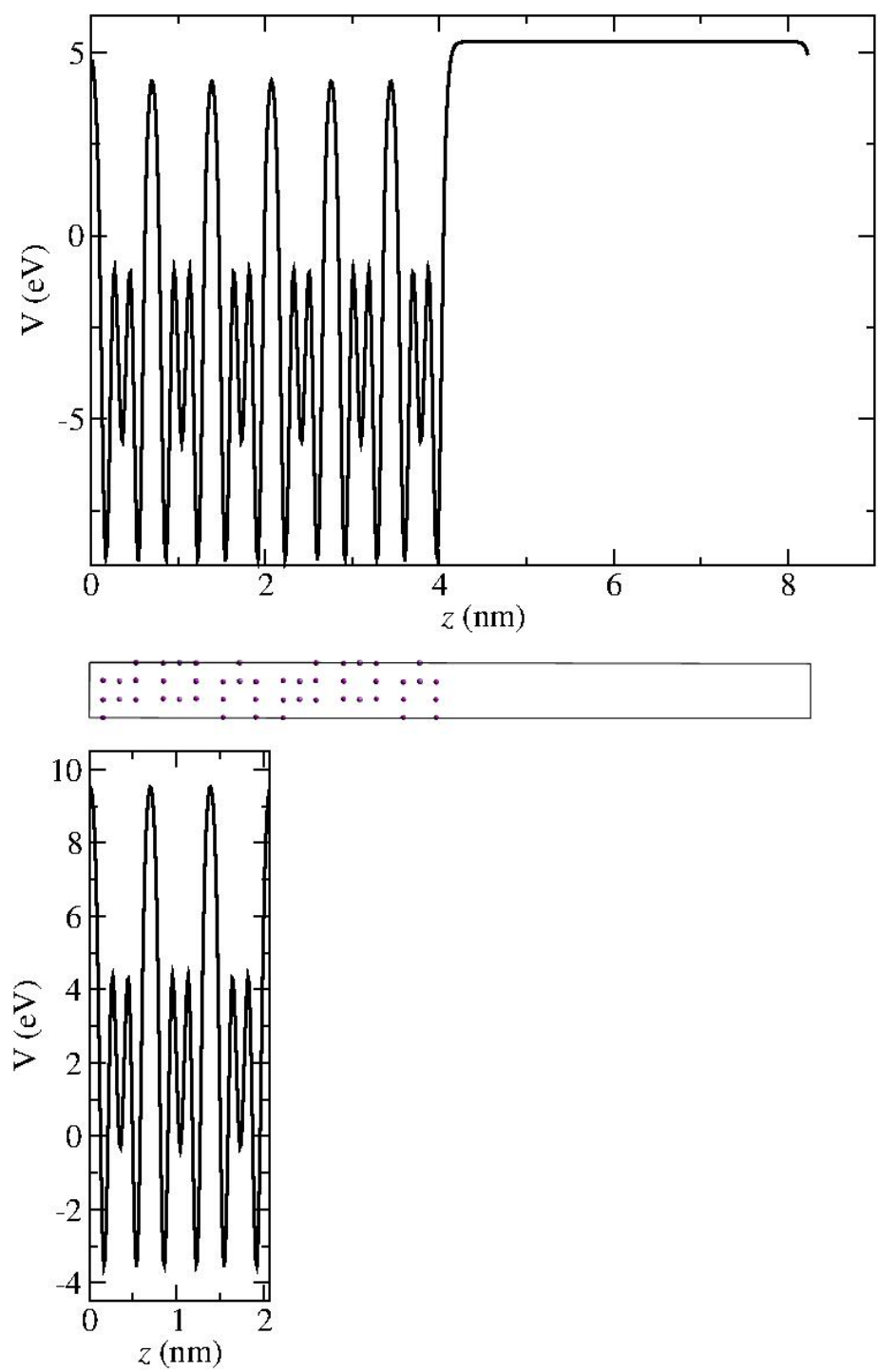

Figure S5. Electronic potential profile of the $\mathrm{BiI}_{3}$-vacuum interface (top panel) and bulk $\mathrm{BiI}_{3}$ material (bottom panel). Atomic structure of the $\mathrm{BiI}_{3}$-vacuum interface (middle panel). 

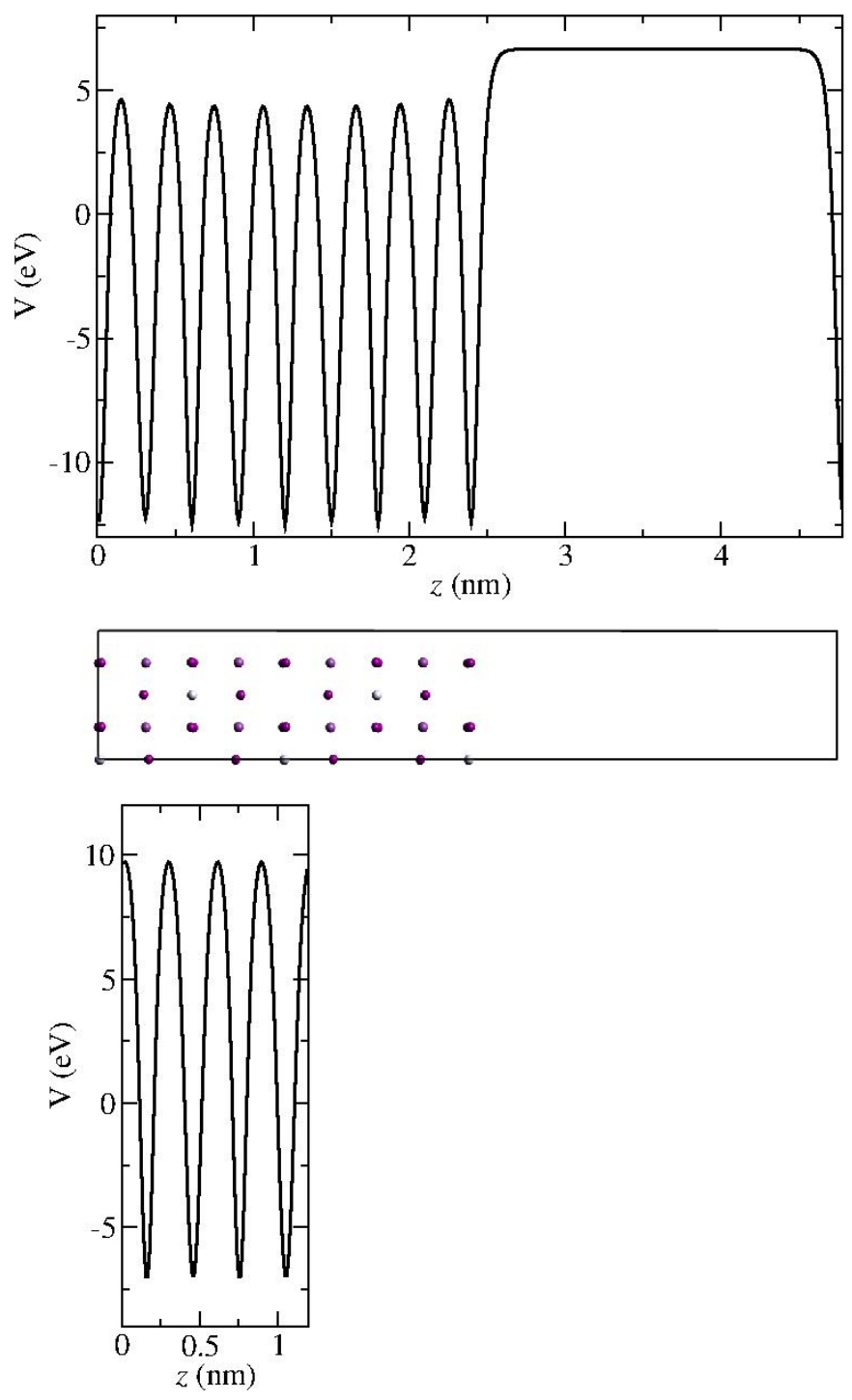

Figure S6. Electronic potential profile of the $\mathrm{AgBiI}_{4}$-vacuum interface (top panel) and bulk $\mathrm{AgBiI}_{4}$ material (bottom panel). Atomic structure of the $\mathrm{AgBiI}_{4}$-vacuum interface (middle panel).

\section{Calculations based on hybrid PBEO functional}

Electronic structure calculations based on the hybrid PBE0 functional were performed using the same geometry that was used in PBE calculations. The same pseudopotentials and kinetic energy cutoffs as in PBE calculations were used. The PBE0 ${ }^{7,8}$ functional was used which mixes the Hartree-Fock and PBE exchange energy in 1:3 ratio and takes PBE correlation energy. The PBE0 functional is known to give significantly better energy gaps and VBM positions than the PBE functional for a variety of semiconducting materials (see for example $\operatorname{Refs}^{9,10}$ ). Calculations were performed using the Quantum Espresso code ${ }^{5,6}$ which implements the adaptively compressed exchange operator approach for the calculation of exchange energy. ${ }^{11} 4 \times 4 \times 4$ grid of points in reciprocal space was used in the calculation. 
To obtain the position of the VBM (and hence the ionization potential) from hybrid functional calculation, we first note that PBE and PBE0 calculations of the same bulk material using the same plane wave computational code have a common energy reference - the average of the electrostatic potential. As a consequence, we obtain the shift of the VBM between PBE and PBE0 calculation simply as the difference of calculated VBMs in the two calculations. We then add this difference to the PBE VBM obtained from the calculation of the interface with vacuum and obtain the PBE0 VBM energy with respect to vacuum. Similar approach for determination of the position of band energies with respect to vacuum was used, for example, in Refs. ${ }^{9,10,12}$

We find that VBM for $\mathrm{AgBiI}_{4}$ and $\mathrm{BiI}_{3}$ are respectively $-6.3 \mathrm{eV}$ and $-6.6 \mathrm{eV}$ when PBE0 functional is used.

We finally discuss the expected differences between the results that would be obtained if $\mathrm{Ag}_{3} \mathrm{BiI}_{6}$ instead of $\mathrm{AgBiI}_{4}$ material were considered in the calculations. Experimental data from Ref. ${ }^{13}$ (inset in Fig. 5c therein) suggest that in the sequence $\mathrm{AgBi}_{2} \mathrm{I}_{7}, \mathrm{AgBiI}_{4}, \mathrm{Ag}_{2} \mathrm{BiI}_{5}$, the VBM increases by about $0.1 \mathrm{eV}$ between each two subsequent elements. Extrapolating this trend to $\mathrm{Ag}_{3} \mathrm{BiI}_{6}$, we can estimate that the $\mathrm{VBM}$ of $\mathrm{Ag}_{3} \mathrm{BiI}_{6}$ would be about $0.2-0.3 \mathrm{eV}$ larger than the VBM of $\mathrm{AgBiI}_{4}$. This correction would put the calculated VBM of Ag-Bi-I even closer to our experimental result. 


\section{REFERENCES}

(1) Lindblad, A.; Söderström, J.; Nicolas, C.; Robert, E.; Miron, C. A Multi Purpose Source Chamber at the PLEIADES Beamline at SOLEIL for Spectroscopic Studies of Isolated Species: Cold Molecules, Clusters, and Nanoparticles. Rev. Sci. Instrum. 2013, 84, 113105. https://doi.org/10.1063/1.4829718.

(2) Perdew, J. P.; Burke, K.; Ernzerhof, M. Generalized Gradient Approximation Made Simple. Phys. Rev. Lett. 1996, 77 (3), 3865-3868.

(3) Hamann, D. R. Optimized Norm-Conserving Vanderbilt Pseudopotentials. Phys. Rev. B Condens. Matter Mater. Phys. 2018, 226 (8), 39-54. https://doi.org/10.1103/PhysRevB.88.085117.

(4) van Setten, M. J.; Giantomassi, M.; Bousquet, E.; Verstraete, M. J.; Hamann, D. R.; Gonze, X.; Rignanese, G. M. The PSEUDODOJO: Training and Grading a 85 Element Optimized NormConserving Pseudopotential Table. Comput. Phys. Commun. 2018, 226, 39-54. https://doi.org/10.1016/j.cpc.2018.01.012.

(5) Giannozzi, P.; Andreussi, O.; Brumme, T.; Bunau, O.; M Buongiorno Nardell; Calandra, M.; Car, R.; Cavazzoni, C.; Ceresoli, D.; Cococcioni, M.; et al. Advanced Capabilities for Materials Modelling with Quantum ESPRESSO. J. Phys. Condens. Matter 2017, 29, 465901. https://doi.org/10.1088/1361-648X/aa8f79.

(6) Giannozzi, P.; Baroni, S.; Bonini, N.; Calandra, M.; Car, R.; Cavazzoni, C.; Ceresoli, D.; Chiarotti, G. L.; Cococcioni, M.; Dabo, I.; et al. QUANTUM ESPRESSO: A Modular and Open-Source Software Project for Quantum Simulations of Materials. J. Phys. Condens. Matter 2009, 21 (39), 395502. https://doi.org/10.1088/0953-8984/21/39/395502.

(7) Perdew, J. P.; Ernzerhof, M.; Burke, K. Rationale for Mixing Exact Exchange with Density Functional Approximations. J. Chem. Phys. 1996, 105 (22), 9982-9985. https://doi.org/10.1063/1.472933.

(8) Adamo, C.; Barone, V. Toward Reliable Density Functional Methods without Adjustable Parameters: The PBE0 Model. J. Chem. Phys. 1999, 110 (13), 6158-6170. https://doi.org/10.1063/1.478522.

(9) Chen, W.; Pasquarello, A. Band-Edge Levels in Semiconductors and Insulators: Hybrid Density Functional Theory versus Many-Body Perturbation Theory. Phys. Rev. B 2012, 86, 035134. https://doi.org/10.1103/PhysRevB.86.035134.

(10) Steiner, K.; Chen, W.; Pasquarello, A. Band Offsets of Lattice-Matched Semiconductor Heterojunctions through Hybrid Functionals and G 0 W 0. Phys. Rev. B 2014, 89, 205309. https://doi.org/10.1103/PhysRevB.89.205309.

(11) Lin, L. Adaptively Compressed Exchange Operator. J. Chem. Theory Comput. 2016, 12 (5), 2242-2249. https://doi.org/10.1021/acs.jctc.6b00092.

(12) Hinuma, Y.; Kumagai, Y.; Tanaka, I.; Oba, F. Band Alignment of Semiconductors and Insulators Using Dielectric-Dependent Hybrid Functionals : Toward High-Throughput Evaluation. Phys. Rev. B 2017, 95, 075302. https://doi.org/10.1103/PhysRevB.95.075302.

(13) Khazaee, M.; Sardashti, K.; Chung, C. C.; Sun, J. P.; Zhou, H.; Bergmann, E.; Dunlap-Shohl, W. A.; Han, Q.; Hill, I. G.; Jones, J. L.; et al. Dual-Source Evaporation of Silver Bismuth Iodide Films for Planar Junction Solar Cells. J. Mater. Chem. A 2019, 7 (5), 2095-2105. https://doi.org/10.1039/c8ta08679f. 